\title{
JÓVENES \\ Y DIVERSIDADES \\ IDENTITARIAS
}

Lola Vázquez Sempértegui

\section{Presentación}

Reflexionar sobre el tema de la nación y la identidad —en particular la identidad juvenil— puede parecer, a simple vista, algo anacrónico. Este tema, que en décadas anteriores tuvo significativa vigencia, hoy es retomado por las nuevas corrientes ideológicas y políticas de América Latina, en las que el Estado-nación es uno de sus ejes fundamentales. La vigencia del Estado tiene su razón de ser, en este momento en el que se está superando la fase más oscura del neoliberalismo, en tanto actor fundamental de las nuevas propuestas políticas.

¿Es posible sostener los Estados-nación sin que se den procesos de recuperación de las antiguas identidades y la construcción de nuevas? No. Por ello, estas nuevas propuestas giran en torno a este eje. ¿De qué otra manera se podría entender la revalidación de las míticas figuras de

* Magíster en Estudios Latinoamericanos. Directora de la Carrera Gestión para el Desarrollo Local. Coordinadora de la Cátedra Monseñor Leonidas Proaño, Universidad Politécnica Salesiana. Docente de la Universidad Politécnica Salesiana. 
Alfaro y Bolívar que ha emprendido el presidente Rafael Correa, o la creación de nuevos símbolos e iconos que intentan reemplazar a los anteriores como es el caso, por ejemplo, de la canción Patria tierra sagrada, que está haciéndose más 'popular' que el Himno Nacional?

Si bien este artículo hace un recorrido histórico sobre las propuestas de construcción del Estado-nación ecuatorianos, el objetivo central de éste es pensar las distintas formas en que la juventud construye sus procesos de identidad particular, en los cuales el Estado es un referente y la identidad nacional deriva en nacionalismo.

Ante procesos de globalización/homogeneización, diversidad/heterogeneidad, la realidad juvenil ha sido vista, en lo académico o lo político, fundamentalmente desde dos posiciones: la una como una memoria del 'ser joven' de décadas anteriores y, por lo tanto, idealizada; y, la otra, como una lectura postestructuralista en la que lo juvenil es analizado desde un relativismo posmoderno.

En este artículo se examinan los alcances de estas dos posiciones y se muestran los límites de cada una de ellas, para llegar a la conclusión de que temas como el Estado-nación, combinados con nuevos referentes como el mercado, el consumo, las nuevas tecnologías, son parámetros de identidad importantes a la hora de mover emociones nacionales y construir identidades particulares en nuestra juventud.

¿Es posible que la juventud viva y sienta lo nacional como un elemento constitutivo de su ser, o son solamente los elementos y las expresiones de la cultura material actual, su único referente identitario? Una pregunta difícil de ser respondida tanto por su complejidad como por el espacio del que disponemos; aquí únicamente avanzaremos ciertas líneas de reflexión y análisis. 


\section{El tema de la identidad}

García Canclini ha expuesto los fundamentos a partir de los cuales se está deconstruyendo el concepto de identidad, teniendo en cuenta que ésta no se constituye sólo en relación con un territorio sino también en su conexión con redes internacionales de mensajes y bienes (léase globalización). Por ello, el autor sostiene el carácter históricamente constituido y, por tanto, no sustancialista de las identidades; y el papel que desempeñan los componentes imaginarios en la constitución de las identidades étnicas y nacionales, así cómo caracterizan éstas las diferencias con otras etnias y naciones.

Así, la identidad no sería la expresión 'natural' de las relaciones que se viven en un territorio, sino la manera en que se imaginan que se viven; la composición multicultural e híbrida de las identidades particulares de cada nación... ${ }^{2}$.

Vista de esta manera, la identidad nacional es un proceso que se constituye en el campo de las relaciones sociales y políticas. Las identidades no existen en forma independiente, se construyen dentro y no fuera del discurso, en ámbitos históricos, instituciones específicas; surgen dentro de modalidades concretas de poder, hay un proceso histórico de constitución de identidades y no una pérdida de identidades; esto sería posible solamente si la vemos como algo esencialista, ontológica e inmutable.

\section{La identidad nacional}

Anderson define a la nación como 'comunidad imaginada', si se ubica este concepto en un contexto histórico, la categoría de 'imaginada' se modificará de acuerdo a cada situación socio-histórica concreta. Por lo tanto, "las 'comunidades imaginadas' tienen que especificar 
quién imagina la comunidad y cómo lo hace, y qué diferencias se suscitan en las comunidades como consecuencia de ello (...) La creación y encarnación de identidades nacionales es, por lo tanto, problemática en el punto de creación [y en] el punto de recepción" (Radcliffe y Westwood, 1999: 50).

De allí que la forma en que se ha imaginado la nación ecuatoriana y quienes la han imaginado han producido imaginarios, referentes y construcciones diferentes, también con implicaciones sociales y políticas.

El Ecuador como Estado-nación ha recorrido un largo camino, y éste se puede analizar a través de sus diversos proyectos. En sus inicios la nación ecuatoriana se inscribe, al igual que el resto de América Latina, en un proyecto de nación dada por la visión y los intereses de los criollos, cuyas formas de imaginarlo han obedecido a intereses específicos y excluyentes, o como dice Vintimilla: "la aristocracia no constituye una clase nacional, la filiación colonial de sus orígenes, la conservación de sus privilegios de casta, y una ideología patriarcal y racista la llevan a elaborar un concepto de nación elitista y excluyente [...] la aristocracia es la esencia y la encarnación material de la nación" (Vintimilla, 1988: 20).

Un segundo momento de formación de la nación ecuatoriana es el que se inicia con la consolidación de una nueva clase social: la oligarquía costeña vinculada a la exportación cacaotera, que rompe con la visión homogénea de nación ecuatoriana creada por los terratenientes serranos; esta nueva clase social origina entonces, según esta autora, un nuevo concepto de nación: una clase social con capacidad de formular un programa político que movilizará otras clases; es decir, otra propuesta de dimensión nacional (ibíd.: 23).

Este proyecto hegemónico nacional, a mediados de los setenta del siglo XX, adquiere nuevas formas con 
los procesos de globalización y neoliberalismo, serán los sectores vinculados al capital financiero los que intenten imponer su idea del Ecuador como Estado 'moderno', mediante un discurso que obedece a la lógica del mercado y en contra de aquellos aspectos que según ellos retrasan el desarrollo del Ecuador, como los requerimientos del pueblo indio o el rol benefactor del Estado. Pero este tipo de Estado-nación, con el tiempo, empieza a ser cuestionado por los nuevos actores sociales que reivindican la descentralización, el respeto a la diversidad y la inclusión social.

Pero estos referentes de constitución de la nación no han sido de una sola vertiente, Vintimilla sostiene que junto al concepto de la nación emblemática, que es igual al interés de una clase convertido en interés nacional, existe la idea del pueblo-nación que tiene un proceso diferenciado del anterior, en tanto este último se configuraría en procesos históricos marcados por las formas que asume la lucha de clases, y que se desenvuelven sobre el fondo de las diversidades étnicas y culturales que revelan la existencia de diversas nacionalidades y rompen la idea de una nación 'monolítica'.

Este análisis tiene una limitación en la medida en que se mira la construcción del Estado ecuatoriano sólo desde el poder y las elites y como algo estático; pero son construcciones sociales que si bien tienen una direccionalidad hegemónica no por ello dejan de participar en ellas otros sectores de la sociedad. En el caso de Ecuador, la creación de la nación ha tenido dos vertientes de constitución: desde un proyecto de los grupos dominantes y desde diversas propuestas de los sectores populares que, a través de procesos de reproducción, resignificación y creación, en un contexto de tensión y conflicto, han participado de una u otra forma en la constitución de la nación y de la identidad nacional ecuatoriana. En este proceso ha 
tenido también una participación importante la juventud. Las movilizaciones estudiantiles tanto en contra de las dictaduras y su lucha por el retorno constitucional como, más tarde, en su participación activa en la caída de Bucaram, Mahuad o Gutiérrez, son algunos ejemplos de ello.

\section{La identidad juvenil}

En este contexto de globalización, de construcción del Estado nacional ecuatoriano, ¿cómo construye la juventud sus formas de identidad?

Existen múltiples versiones sobre la forma en que los jóvenes estarían construyendo su identidad en esta nueva época. Para algunos autores (Lyotard, Maffessoli,) estamos ante una nueva época cultural llamada, en términos genéricos, posmoderna; otros (Giddens, por ejemplo) plantean que estamos más bien en una fase de radicalización de la modernidad. De cualquier forma, el hecho es que se vive un cambio cultural profundo, ya no sólo a nivel local sino a escala mundial, que estaría produciendo modificaciones en las formas de conocer, de percibir el mundo; formas de institucionalidad y de normatividad que están influyendo decisivamente en las concepciones y prácticas de la juventud de hoy.

El concepto de juventud (que en este trabajo tiene una connotación urbana) es relativamente simple si éste se refiere a un cierto periodo de edad, generalmente entre 14 y 30 años (según la Ley de la Juventud aprobada en el Ecuador). Sin embargo, la definición se vuelve mucho más compleja si se incorporan elementos culturales a la misma, ya que muchas de las características que se atribuyen a la juventud corresponden a determinaciones culturales; por lo que se trataría de rasgos que se refieren a ciertas sociedades, épocas o visiones más particulares y 
que no necesariamente se reproducirían en los jóvenes en forma absoluta.

Desde diferentes posiciones se ha impuesto un modelo de aquello que los jóvenes deberían ser: idealistas, renovadores, comprometidos con el cambio social; como no siempre sus prácticas están dentro de esos referentes, entonces se los enjuicia y se los sentencia. Juzgamiento que nace al no entender a la juventud como resultado de procesos socio-históricos específicos, y al medir a la generación actual con los mismos parámetros de generaciones anteriores. En los espacios tanto públicos como privados hay una tendencia a estigmatizar a la generación actual basados en valores cívicos, éticos y morales de generaciones pasadas.

De allí la necesidad de acudir a los referentes históricos para llevar adelante procesos sociales diferentes que se acerquen con otra mirada a la juventud de hoy. Es necesario analizar esta generación con los valores, patrones conceptuales y analíticos de este periodo. Solamente caracterizando la época actual es que se podría analizar a la juventud de este tiempo y entender cuáles son sus percepciones sobre los diversos aspectos que atañen a su vida.

Marcelo Urresti (2000: 177) señala que la comparación de las generaciones de los años sesenta y setenta con las de los ochenta y noventa (y no se diga con la de 2010) funciona como una suerte de rasero, en donde el punto de comparación es lo que le sobra o le falta a la generación actual en relación a la anterior. Pero lo que interesa ubicar es que se trata de épocas históricas que definen los temas y la manera de ser de forma diferente, por lo que la vivencia y la experiencia social también son diferentes.

Así, es común en la literatura y en los análisis sobre la juventud atribuir a la generación de los sesena y setenta - en el contexto de su época- un compromiso 
social y político, mientras se atribuye a la actual precisamente lo opuesto, poca sensibilidad social. "Para decirlo con una frase ilustrativa, no es que los jóvenes de hoy son consumistas y los de los años sesenta politizados. En los años sesenta era tan improbable tener afinidades alejadas de la política como hoy su contrario, y esto no tiene que ver sólo con los jóvenes" (Ibíd., 178). Es ante todo un hecho que tiene que ver con el conjunto de la sociedad. Por lo tanto, entender a los jóvenes de hoy pasa por comprender el contexto de la época y la generación que les ha tocado vivir.

La generación de los años sesenta y setenta vivió en un contexto social, tecnológico, económico y cultural totalmente diferente al actual; por ello, sus comportamientos, compromisos y expectativas eran notablemente distintos respecto a los de ahora; vivieron una época histórica que hablaba de otra forma de ser y estar en el mundo, de vivir sus dimensiones, en relación con códigos, estructuras del sentir y de pensar distintas.

Las sociedades funcionaban con un modelo económico que, aunque injusto, tenía una concepción social más integradora. La actividad productiva tenía como objetivo fundamental la rentabilidad sustentada en la producción en masa, para la que ocupaba abundante materia prima y mano de obra, lo que generaba estabilidad laboral y mayores certezas de futuro en la juventud, como la de conseguir un empleo y mantenerlo, ya que generalmente era de por vida.

El Estado cumplía funciones diferentes a las de los años ochenta y noventa; su papel era decisivo en el manejo de las economías: intervenía, orientaba, controlaba los procesos productivos, generaba fuentes de trabajo; lo que provocó el aumento de la burocracia y de la clase media. La escuela y la educación cumplían un papel importante en los procesos de socialización, de ascenso 
social, de mejores oportunidades. Las industrias culturales también tenían un perfil que, directa o indirectamente, apoyaba esta dinámica. La televisión no tenía el impacto de hoy: ... "cimentaba cierta idea del 'nosotros nación' por detrás de su funcionamiento" (ibíd., 183). Cumplía, entonces, una función congregante y uniformizadora.

Los años sesenta son aquellos en los que se difunde y consolida por primera vez un conjunto de expresiones culturales (que por acción de las industrias se generalizarían como consumos masivos), como el rock, que se manifestaban en estilos de vida, lenguajes, y que tenían como objetivo marcar una frontera con el mundo adulto.

Era una época de profunda politización, el conflicto entre comunismo y capitalismo determinará el comportamiento de los Estados y los ciudadanos, pero también el de la juventud. Se generaba un imaginario de los jóvenes como 'rebeldes', 'luchadores', 'revolucionarios', que tenía su sustento en la activa participación en procesos revolucionarios y de resistencia en todo el mundo, como en Vietnam, Angola, Cuba, en los movimientos estudiantiles y juveniles de Tlatelolco, el Cordobazo y el Mayo francés. En conclusión, era una época de ascenso y participación política de las sociedades, que 'contaminaban' también a los jóvenes -aunque no a todos-, haciendo socialmente visibles las formas de participación política.

La idea de nación estaba representada por los sueños de constitución de la Gran patria latinoamericana y la oposición a la nación-imperio. La denuncia del imperialismo y del proceso de dependencia, desarrollada desde la CEPAL, entre otros sectores, levantó toda una consigna antiimperialista. La juventud se alineó en estas posiciones a lo largo de América Latina y, en ella, Ecuador. 
Estos procesos se convirtieron en una corriente cultural decisiva en la conformación de identidades sociales de la generación de los sesenta y setenta. El referente estudiante tenía una connotación política que generaba patrones políticos y culturales para los jóvenes y el conjunto de la sociedad. Era una generación que se miraba a sí misma y era mirada por otros sectores de la sociedad como portadora de ideas de transformación social y política. Los años sesenta y setenta fueron los años de los grandes movimientos estudiantiles.

Esta situación empezó a cambiar durante los años ochenta. La crisis de la deuda externa que impactó a América Latina y Ecuador generó una situación de crisis, en la que la percepción de 'seguridad' económica dejó de ser tal y dio paso a una época de inestabilidad económica, social y política. En el país fue el inicio del neoliberalismo, modelo que modificará sustancialmente las formas económicas y los roles y funciones de las instituciones políticas y sociales.

Muchas de las ideas de transformación social no lograron concretarse en las generaciones de los ochenta y noventa. Los movimientos estudiantiles comenzaron a despolitizarse y a predominar intereses ajenos a los discursos políticos; las condiciones sociales sufrieron fuertes cambios en las formas de participación y asociación, los jóvenes empezaron a luchar por su supervivencia. El paradigma tradicional a través del cual se configuraba el mundo juvenil estaba prácticamente determinado por lo político, y se tornó inoperante el momento de abordar una nueva realidad juvenil caracterizada por su heterogeneidad.

Este proceso se radicalizó en las dos últimas décadas y fue inscrito entre las grandes transformaciones mundiales. Un gran desarrollo de la tecnología, en espe- 
cial de la informática y las comunicaciones, reordenó la vida social y la cotidianidad, la realidad se fragmentó y se complejizó, en especial en cuanto a las dinámicas económicas, a las de participación y organización, que rompió con formas y dinámicas anteriores.

A nivel de la producción los cambios fueron sustanciales, de un modelo de producción en masa que primó en décadas anteriores se pasó a procesos productivos reprogramables que limitan la producción en serie, volviendo las mercancías más individuales'.

Los Estados, convertidos en el centro de los ataques del neoliberalismo, tuvieron que reorientar su función y comenzar un proceso de subasta de sus recursos a través de la privatización, se redujeron las inversiones en áreas consideradas estratégicas (salud, educación), disminuyó la oferta de empleo desde el Estado. Es así como estos fueron focalizando sus intervenciones, desentendiéndose de la gestión de la vida de la población, llegando, finalmente, a limitarse a arbitrar conflictos entre partes, contribuyendo, cuando lo hizo de manera deficiente, a extender el clima de desigualdad social que, progresivamente, se radicalizó.

El empleo fue cambiando: de ser un derecho pasó a convertirse en un privilegio, lo que generó cambios en las percepciones de los jóvenes: quienes ya no parten de 'seguridades' viven un proceso de incertidumbre ante el futuro. Es una situación completamente distinta a las décadas anteriores; hoy estamos frente a procesos de desestructuración social, fragmentación económica y política.

Existe una gran expansión de la industria cultural y las comunicaciones, que traspasa las fronteras nacionales provocando procesos de homogeneización cultural a nivel mundial; sin embargo, esta homogeneidad no es 
masiva, este proceso se da en sectores de las sociedades que tienen acceso a esta industria cultural. Aunque hay producción cultural masiva de un lado, del otro se da una segmentación del mercado creando consumidores exclusivos para ciertos productos. Como dice Urresti: "Los consumos de tiempo libre y las tecnologías disponibles para facilitarlo tienen inscripta la doble lógica de la privatización y la especificación. Ante todo, son tecnologías audiovisuales las que prácticamente monopolizan el tiempo libre de la mayoría de la población. Esto nos habla de un profundo cambio en la cultura" (ibíd.: 192).

Es decir, los medios masivos de producción de cultura modifican la realidad; así, por ejemplo, la televisión ya no refleja las realidades locales y nacionales como en décadas pasadas, sino que se pasa de la idea de comunidad-nación a una idea de comunidad global. Regiones y países del mundo, antes aislados y desconocidos, se acercan gracias a la tecnología, que modifica los ritmos y los tiempos locales para rearticularse a ritmos y tiempos globales. "La ruptura de los tiempos históricos implica, entre otras cosas, la rearticulación de la memoria colectiva y de los relatos personales, es decir, de las formas culturales dentro de las que se construye la identidad y se reconoce a los semejantes. Esto contribuye a la obsolescencia y alteración de formas simbólicas de la vida social" (ibíd.: 194).

Este período caracterizado por la crisis y la recesión, por las políticas de ajuste neoliberal, por fenómenos trans-territoriales masivos y por una producción cultural global, va acompañado de la pérdida de la credibilidad de las instituciones tradicionales como el Estado, la familia, los partidos políticos, y la participación política va perdiendo referencialidad.

En este contexto, nuevas figuras juveniles con nuevos referentes, fundamentados sobre todo en la música, cobran fuerza y visibilización social. La juventud se ha 
alejado de los metarrelatos y empieza a identificarse más con micro-discursos, con identidades particulares. Muchas de éstas han sido recogidas en el análisis social como las 'tribus urbanas' de Maffesoli (1990), que se articulan en torno a intereses más particulares y específicos como grupos musicales, deportivos. O como 'culturas juveniles', según Carles Feixa, para quien éstas "se refieren a la manera en que las experiencias sociales de los jóvenes son expresadas colectivamente mediante la construcción de estilos de vida distintivos, localizados en el tiempo libre, o en espacios intersticiales de la vida institucional" (Feixa, 1998: 84).

Es decir, la manera en que las experiencias sociales de los jóvenes son expresadas colectivamente mediante la construcción de estilos de vida distintivos, con grados significativos de autonomía respecto del mundo adulto. Vale mencionar, no obstante, que si bien una de las formas importantes de identidad son estas culturas juveniles, éstas no son las únicas ni toda la juventud se adscribe a ellas.

Hay gran diversidad de formas de expresión de las manifestaciones culturales juveniles, buena parte de los estudios sobre el tema se refieren a aquellas que asoman como más 'espectaculares', como el rock, el punk, o expresiones más violentas como las 'pandillas', estudios que en ocasiones se quedan en la forma y pierden de vista la dimensión política y de sentido de vida de las mismas.

La principal forma de expresión de los jóvenes actuales ya no está dada por el discurso sino por la simbología, de allí que cobra gran importancia la estética, un determinado estilo de vestir, o de adorno, que otorgan sentido y dan pertenencia. Pero las culturas juveniles, como expresiones culturales de los jóvenes, son espacios en donde también se manifiesta una postura de contra- 
dicción y oposición de profundo contenido político (Vázquez, 2001: 82 y ss.). que se evidencia de múltiples formas, una de ellas es la ruptura con tipos de organización más rígidas y 'salvadoras' como las de décadas anteriores, representadas en grupos políticos partidistas y de la Iglesia.

La globalización desestabiliza muchos de los referentes materiales y simbólicos de las identidades colectivas de épocas pasadas. A las clases sociales, basadas en intereses económicos, se sobrepone una multiplicidad de intereses y representaciones de múltiples actores sociales que reivindican su identidad desde la diferencia, pasando de una identidad fundamentada en la razón, a otra en torno a emociones, símbolos y gustos.

El panorama social ha cambiado y con él se ha transformado también la identidad o la forma cómo se perciben y definen los jóvenes. Varios de los estudios posmodernos sobre el tema juvenil ubican al mercado, al consumo y a la cultura de la imagen ${ }^{3}$ como los nuevos referentes de construcción de identidad.

Con respecto al mercado se dice que en esta época es el principal regulador de las relaciones sociales, en tanto integra simbólicamente a los diferentes sectores en el consumo. El consumo como comportamiento se instala en las nuevas generaciones como dispositivo de integración. El consumo aparece como un elemento importante en la formación de las identidades juveniles. Los jóvenes saben que de acuerdo a los elementos que consumen (ropa, tipo de música) definen no sólo el nivel socioeconómico al que están adscritos sino, también, a la cultura juvenil con la que se identifican. Existiría, por lo tanto, un fundamento cultural frente al comportamiento consumista y estaría relacionado con el tema de la imagen o la expresión a través del cuerpo, en gestos, movimientos, 
tatuajes, que muestran a los demás todo lo que piensan y sienten, sin mediaciones discursivas.

Pero esta visión no es absoluta ni homogénea, los jóvenes acceden al consumo desde una posición de confrontación/negociación. A simple vista parece que la industria cultural homogeneiza los gustos, la moda y el consumo y que no hay diferenciación entre los jóvenes; muchos de los análisis se inscriben en esta línea y ven a la juventud como simple imitadora de pautas de consumo masivo, no se toman en cuenta los procesos de resignificación que hacen, de acuerdo a su experiencia, de sus concepciones de vida y situación económica. La homogeneización se da más bien por arriba, es decir, en los sectores ricos de la sociedad; pero habría que preguntarse: ¿Cuántos jóvenes se quedan por fuera de este circuito comercial?, o ¿de qué forma van accediendo, aunque sea desde los márgenes, a esta cultura globalizada? Los jóvenes de sectores medio altos y altos pueden satisfacer esos patrones de consumo, pero para la juventud empobrecida de nuestro continente es una realidad virtual y un mundo mágico trasmitido por la televisión, que muestra una sociedad de consumo que no compagina con su realidad cotidiana (Vázquez, 2001: 82 y ss.).

Con estos referentes los jóvenes de esta generación construyen su identidad alejados de las formas institucionales y de socialización características de la generación anterior: el Estado, la familia, la Iglesia; la construyen desde otros espacios, desde otros referentes como los medios de comunicación, las industrias culturales, los grupos de pares, que tienen a su vez otras expresiones organizativas, ya no son los grupos políticos, deportivos o religiosos los más significativos, ahora lo constituyen la jorga, la banda, el grupo de esquina. La causa, las ya señaladas: la pérdida de referentes institucionales y la descon- 
fianza en los partidos políticos, que copan la noción de política divorciada de la vida cotidiana y que generan un horizonte poco atractivo para la participación de los jóvenes.

\section{Retomando el tema del Estado-nación y la identidad nacional}

Autores como Kellner (cit. por Jorge Larraín, op. cit. 112), plantean que la identidad es un juego mediante el cual las personas optamos por cualquiera de ellas, la característica de este tiempo sería precisamente la mayor cantidad de opciones que la gente tiene para cambiar de identidad. Larraín cuestiona a Kellner diciendo que es una exageración, en tanto no existe la tan mentada libertad para elegir y cambiar de identidad como quien se cambia de ropa. Larraín dice: "El problema está en que Kellner parece entender por identidad la mera apariencia externa (...) uno puede cultivar una imagen, pero esto no siempre toca los aspectos más básicos de la identidad" (ibíd.: 112-113). Este tema tiene que ver con uno de los problemas de los estudiosos de lo juvenil, y que es precisamente sobredimensionar lo externo, la moda, para atribuir identidad y, de allí, que se les asigne a los jóvenes una serie de identidades atendiendo sólo a su forma de vestir o al uso de accesorios.

Lo señalado permite concluir que no existe una identidad juvenil, sino unas identidades juveniles diferenciadas, que se moldean según las experiencias individuales y sociales, por lo tanto, no se pueden hacer generalizaciones; si bien existen parámetros comunes: la pertenencia a un país, una historia común, una lengua y hasta una religión más o menos homogénea; también hay una serie de elementos que los diferencian: procedencia económica y social, adscripción o no a una cultura juvenil. 
Desde la primera década del siglo XXI, un viejo referente de constitución identitaria para la juventud, el Estado-nación, cobra nuevamente importancia. Se han realizado propuestas de reconstitución de los Estados en América Latina y en particular en Ecuador. Esto significa que se retoma el tema de la institucionalidad y lo público desde nuevos parámetros. Quizá uno de los más importantes el del Estado plurinacional, que significa como pensar una nueva forma de construir un Estado más democrático e incluyente no solamente desde lo idígena, sino lo mestizo, lo afro y lo montubio.

Este Estado va a ser fundamental a la hora de construir identidades, la identidad mestiza como hegemónica en el mundo juvenil urbano va a ser interpelada y cuestionada desde los que, hasta ese entonces, constituían 'los otros', los indígenas.

La propuesta del estado ecuatoriano no tiene una definición clara de las políticas sobre la juventud, pero le ha dado formas de incorporación institucionales y ésta ha encontrado en el periodo nuevos mecanismos de inclusión y participación. Si bien no se cuenta con datos que avalen la afirmación de que el proceso de la Revolución Ciudadana cuenta con un contingente juvenil importante, nos remitimos a la observación; a través de ella se ha constatado la presencia numerosa de jóvenes en los procesos de campaña electoral, particularmente en torno a la aprobación de la Constitución de 2008, participación dada en el nivel de propuestas y de movilización social; ha participado también en el debate y la acción social en torno a diversos temas nacionales en los que la juventud parecía no interesarse más.

Todos estos elementos están configurando un nuevo joven con identidad nacional, que no está dada solamente por la propuesta del gobierno, sino por otros 
referentes como la solidaridad latinoamericana, la presencia ecuatoriana en el deporte mundial, que dotan a los jóvenes de esta generación de parámetros de identidad antiguos, pero resignificados por otros valores y otras prácticas.

\section{Concluyendo}

La juventud actual construye sus formas de identidad juvenil, nacional, étnica, de género y etárea propias bajo condiciones nacionales y mundiales diferentes. Los procesos de globalización, los grandes movimientos migratorios, entre otros, generan nuevos imaginarios simbólicos, sociales y culturales. Por lo tanto, la juventud resume en su ser e identidad las tensiones, conflictos y paradojas que vive la sociedad actual y que están modificando sus formas de conocer y de percibir el mundo.

Hoy los jóvenes interpelan al mundo adulto sobre la veracidad de su discurso sobre el Estado, la educación, la familia, la religión, que no siempre coincide con la práctica.

Buscan espacios de participación en el Estado y sienten que pueden ser portadores de un proyecto de presente y de futuro. De tal forma que los jóvenes comienzan a mirarse a sí mismos como portadores de otro tipo de nación.

La juventud es un grupo social presente, actual, con todas sus potencialidades, que puede incorporarse en las propuestas de construcción de las nuevas formas de Estado y de nación que surgen en América Latina; así como pueden participar con su voz en la Academia interpelando con sus nuevas formas de ser y de aprender, e interpelando a la Iglesia para que ésta se acerque a ellos y no al revés. 


\section{Notas}

1 Algunas ideas de este artículo fueron trabajadas en la Tesis $L a$ nación ecuatoriana desde los jóvenes. Percepciones de los jóvenes y las jóvenes de Quito sobre la nación y la identidad nacional. Lola Vázquez. Universidad Andina Simón Bolívar. Área de Estudios Sociales y Globales. Programa de Maestría en Estudios Latinoamericanos. Mención en Estudios de la Cultura. 2002.

2 García Canclini, citado en Nelly Arenas, Globalización, integración e identidad: América Latina en las nuevas perspectivas, Caracas, Centro de Estudios para el Desarrollo (Cendes), s.f.

3 Revista Occidente $\mathrm{N}$ : 4, Jóvenes: del mito vanguardista a una nueva identidad juvenil, Año LVI, enero-marzo 2002, versión electrónica.

\section{Bibliografía}

ANDERSON, Benedict

1993 Comunidades Imaginadas, México: Fondo de Cultura Económica.

ARENAS, Nelly

s/f Globalización, integración e identidad: América Latina en las nuevas perspectivas, Venezuela, Centro de Estudios para el Desarrollo (Cendes).

CERVIÑO, Mauro, Cinthia Chiriboga y Carlos Tutivén

2000 Culturas juveniles, cuerpo, música sociabilidad \& género, Guayaquil, Ediciones Abya-Yala-Convenio Andrés Bello.

FEIXA, Carles

1998 El reloj de Arena. Culturas Juveniles en México, México: Centro de Investigación y Estudios sobre Juventud.

LARRAÍN, Jorge

1996 Modernidad, razón e identidad en América Latina, Santiago de Chile: Editorial Andrés Bello.

MAFFESOLI, Michael

1990 El tiempo de las tribus: el declive del individuo en las sociedades de masas, Barcelona: Icaria.

RADCLIFFE Sarah y Sallie Westwood

1999 Rehaciendo la Nación. Lugar, identidad y política en América Latina, Quito: Ediciones Abya-Yala. 
URRESTI, Marcelo

2000 "Paradigmas de participación juvenil: un balance histórico", en La participación social y política de los jóvenes en el horizonte del nuevo siglo, Buenos Aires: Gráficas y Servicios, SRL, 177-206, 2000.

VÁZQUEZ, Lola

2001 "Culturas Juveniles", en Acerca de Jóvenes, contraculturas y sociedad adultocéntrica, Klaudio Duarte y Danahé Zambrano, ed, San José, Costa Rica, Editorial Departamento Ecuménico de Investigaciones (DEI), 75-90.

VINTIMILLA, María Augusta

1988 "Proyecto Histórico, nación y cultura", en Estado, nación y cultura. Los proyectos históricos en el Ecuador, Cuenca: Universidad de Cuenca, Instituto de Investigaciones Sociales (IDIS), 1988. 